\title{
Hiperplasia endometrial asociada al uso del tamoxifeno en pacientes con cáncer de mama en Barranquilla
}

Endometrial hyperplasia associated with use of tamoxifen in patients with breast cancer in Barranquilla

\begin{abstract}
María Hemer-Cera', Ricardo Donado-Botero', Carmen Barcasnegras-Deavila', Andrés García-Osorio', Daniel Turcios-Ordoñez', Ángel Hernández-Lastra², Martha Peñuela Epalza ${ }^{3}$ CvLAC, Víctor Floréz-García ${ }^{3^{*}} \otimes$ CvLAC
\end{abstract}

Fecha correspondencia: Recibido: enero 2 de 2020. Revisado: enero 19 de 2021. Aceptado: enero 23 de 2021.

Forma de citar: Hemer-Cera M, Donado-Botero R, Barcasnegras-Deavila C, García-Osorio A, Turcios-Ordoñez D, Hernández-Lastra A, et al. Hiperplasia endometrial asociada al uso del tamoxifeno en pacientes con cáncer de mama en Barranquilla. Rev CES Med. 2021; 35(1): 16-25.

Open access

(c) Derecho de autor

Licencia creative commons

Ética de publicaciones

Revisión por pares

Gestión por Open Journal System DOl: http://dx.doi.org/10.21615/ cesmedicina.35.1.2

ISSN 0120-8705

e-ISSN 2215-9177

Comparte

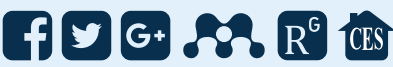

\section{Resumen}

Introducción: el tamoxifeno ha sido el medicamento de primera línea para el tratamiento del cáncer de mama; sin embargo, se han evidenciado serios eventos adversos con su uso. El objetivo de este trabajo fue determinar la hiperplasia endometrial asociada al uso de tamoxifeno en mujeres con cáncer de mama en una institución oncológica de la ciudad de Barranquilla- Colombia. Metodología: estudio de casos y controles retrospectivo. Se incluyeron 202 pacientes con cáncer de mamá tratadas entre 2012 y 2017 . Fueron evaluadas variables antropométricas, sociodemográficas, personales, clínicas y patológicas. Se estimaron los Odds Ratios (OR) crudos y ajustados. Resultados: 68 participantes fueron diagnosticadas con hiperplasia endometrial secundaria al tratamiento antineoplásico. De éstas, 59 (86,7 \%) usaron tamoxifeno, la mayoría (37,2 \%) por un lapso de 6-11 meses. El análisis bivariado mostró asociación entre la hiperplasia endometrial y uso del tamoxifeno con un OR de 3,9 (IC95 \%: 1,8-8,5) y 2,9 (IC95\%: 1,18-7,5) en los análisis crudos y ajustados, respectivamente. Conclusión: el uso de tamoxifeno se asocia con la presencia de hiperplasia endometrial.

Palabras clave: Tamoxifeno; Cáncer de mama; Complicaciones, Hiperplasia endometrial.

\footnotetext{
Abstract

Introduction: tamoxifen has been the first line drug for the treatment of breast cancer; however, serious adverse events have been evidenced with its use. The aim of this study was to determine endometrial hyperplasia associated with the use of tamoxifen in women with breast cancer in an oncological institution in the city of Barranquilla, Colombia. Methodology: retrospective case-control study. A total of 202 patients with breast cancer treated between 2012 and 2017 were included. Anthropometric, sociodemographic, personal, clinical and pathological variables were evaluated. Crude and adjusted Odds ratios (OR) were estimated. Results: 68 parti- cipants were diagnosed with endometrial hyperplasia secondary to antineoplastic treatment. Of these, 59 (86.7 \%) used tamoxifen, the most of them (37.2 \%) for a period of 6-11 months. Bivariate analysis showed
} 


\author{
Sobre los autores: \\ 1. Estudiante de Medicina, \\ Universidad del Norte. \\ Barranquilla (Colombia). \\ 2. Especialista en \\ Hematología y Oncología \\ Clínica, Organización \\ Clínica Bonnadona Prevenir. \\ Barranquilla (Colombia). \\ 3. Profesor. Departamento \\ de Salud Pública, División \\ de Ciencias de la Salud, \\ Universidad del Norte. \\ Barranquilla (Colombia).
}

El tamoxifeno es el medicamento de elección para el cáncer de mama, dadas sus propiedades antagónicas; sin embargo, tiene un efecto estrogénico sobre el endometrio y se ha reportado su asociación con cáncer endometrial de bajo grado en mujeres posmenopáusicas. an association between endometrial hyperplasia and tamoxifen use with an OR of 3.9 (95\% Cl: $1.8-8.5)$ and $2.9(95 \% \mathrm{Cl}: 1.18-7.5)$ in the crude and adjusted analyses, respectively. Conclusion: tamoxifen use is associated with the presence of endometrial hyperplasia.

Keywords: Tamoxifen; Breast cancer; Complications; Endometrial hyperplasia.

\section{Introducción}

El cáncer de mama en la población colombiana se ha convertido en un verdadero reto para el sistema de salud. Se estima que en 2018 Colombia presentó cerca de 13380 casos nuevos de cáncer de mama con una tasa estandarizada de 44,1 por 100000 mujeres, convirtiéndose en el primer cáncer entre las mujeres colombianas (1).

Para el tratamiento avanzado, el tamoxifeno es el medicamento de elección dadas sus propiedades antagónicas; sin embargo, tiene un efecto estrogénico sobre el endometrio, que amerita su estrecha vigilancia, puesto que se ha reportado su asociación con cáncer endometrial de bajo grado en mujeres posmenopáusicas (2). Aun teniendo en cuenta esta relación y algunos otros efectos adversos frecuentes (3), sigue siendo de primera línea para el tratamiento del cáncer de mama debido a distintos factores, entre ellos su costo-efectividad y consideraciones positivas respecto al riesgo-beneficio, demostrando reducción del riesgo de recurrencia (34 \% - 51 \%) y mortalidad (24 \%-37 \%) (4).

Entre los factores que favorecen la aparición de hiperplasia endometrial se ha descrito un índice de masa corporal aumentado, síndrome de ovarios poliquísticos, terapia de reemplazo hormonal sistémica, diabetes, infertilidad e hipertensión. Otras condiciones predisponentes son la postmenopausia, nuliparidad, menopausia tardía, menarquía temprana y anovulación crónica (5-7).

Desde esta perspectiva, algunos autores proponen que aunque la supervivencia es el resultado buscado, no siempre se obtiene bajo los mejores escenarios dado el impacto en la calidad de vida del paciente y el deterioro en la calidad de vida de su núcleo familiar (8-10). Específicamente, en mujeres con hiperplasia endometrial se espera que estas se sometan a un tratamiento para esta nueva comorbilidad, bien sea progestágenos tanto orales como locales (sistema intrauterino liberador de levonorgestrel), ya que son eficaces para lograr la regresión de la hiperplasia endometrial sin atipia; mientras que en aquellas que la hiperplasia es atípica se ha recomendado la histerectomía total debido al riesgo de malignidad o progresión a cáncer (6).

El objetivo del presente estudio fue evaluar la relación de hiperplasia endometrial con el uso de tamoxifeno en pacientes con cáncer de mama en un centro de referencia oncológico de la ciudad de Barranquilla.

\section{Materiales y métodos}

Se realizó un estudio observacional analítico retrospectivo tipo casos y controles no pareados. La variable independiente fue definida como presencia o ausencia de hiperplasia endometrial posterior al inicio del tratamiento con tamoxifeno para cáncer de mama. Todas las participantes de este estudio tenían al menos 18 años. Los casos fueron definidos como pacientes con cáncer de mama como primer diagnóstico e hiperplasia endometrial diagnosticada mediante ecografía transvaginal posterior al inicio del tratamiento utilizado para combatir el cáncer de mama. 
Enero - abril de 2021 - Pág 18

La hiperplasia endometrial con atipias fue mayor entre las usuarias de tamoxifeno a largo plazo.
La definición de controles estuvo dada como pacientes con cáncer de mama como primer diagnóstico y no haber cursado con hiperplasia endometrial durante el tiempo posterior al inicio de tratamiento contra el cáncer de mama.

Con el fin de evaluar el periodo exacto de exposición, en la mayoría de los casos se encontró la fecha exacta del final del tratamiento; sin embargo, cuando no se pudo definir con certeza, se usó la última fecha reportada en la historia clínica donde se menciona el tratamiento. En las pacientes que recibieron el tratamiento en periodos no consecutivos, el tiempo total de uso se calculó sumando dichos períodos. Se excluyeron del estudio mujeres que previamente al inicio del tratamiento tuvieran hiperplasia endometrial o se les hubiera realizado histerectomía.

Se realizó un análisis descriptivo según la naturaleza cualitativa de las variables de estudio. Para evaluar la relación del tratamiento con tamoxifeno y la hiperplasia endometrial se calcularon la odds ratio (OR) y los intervalos de confianza (IC) del $95 \%$ y se realizaron pruebas de tendencia. Se aplicó la prueba Chi-cuadrado o la prueba exacta de Fisher para el análisis bivariado. Posteriormente, se realizó un análisis multivariable tipo regresión logística multivariada incondicional; fueron considerados significativos valores de $p$ menores o iguales a 0,05. Todos los análisis estadísticos fueron realizados usando SPSS v. $25^{\circledR}$.

Este estudio se acoge a la normatividad sobre investigación con seres humanos y fue presentado y aprobado por el Comité de Ética de la Universidad del Norte.

\section{Resultados}

Fueron incluidos 68 casos y 134 controles. La mayoría de las participantes eran multíparas, de 48 años o mayores, posmenopáusicas, con un índice de masa corporal en rango de normalidad, de estrato socioeconómico bajo (estratos 1 y 2) y trabajadoras. El fenotipo del cáncer de mama predominante fue el luminal A (en $75 \%$ de los casos y $46,3 \%$ de los controles), siendo el estadio II el mas predominante en ambos grupos (cuadro 1).

De las 202 mujeres estudiadas, 33,7 \% fueron diagnosticadas con hiperplasia endometrial secundaria al tratamiento antineoplásicos; de éstas, 59 (86,7 \%) usaron tamoxifeno, la mayoría (37,2 \%) por un lapso de 6-11 meses conllevando a una hiperplasia sin atipias en el 59,1\%. Nueve de los 68 casos (13,2 \%) no usaron el medicamento.

La hiperplasia endometrial con atipias fue mayor entre las usuarias de tamoxifeno a largo plazo (53,3 \% en las usuarias de 2 -3 años) que, en las no usuarias, en quienes la hiperplasia sin atipias fue la única presente; sin embargo, la hiperplasia sí generó incapacidad en la mitad de las usuarias del medicamento de $4-\geq 5$ años (50 \%) (cuadro 2). 
El $86,7 \%$ de los casos y el $62,7 \%$ de los controles, usaron tamoxifeno, encontrándose asociado a hiperplasia endometrial (OR=3,90; IC95\%: 1,781-8,55)
Cuadro 1. Descripción sociodemográfica de casos y controles

\begin{tabular}{|c|c|c|c|c|}
\hline \multirow[b]{3}{*}{ Características } & \multirow{2}{*}{\multicolumn{2}{|c|}{$\begin{array}{c}\text { Casos } \\
n=68\end{array}$}} & \multirow{2}{*}{\multicolumn{2}{|c|}{$\begin{array}{c}\text { Controles } \\
n=134\end{array}$}} \\
\hline & & & & \\
\hline & $n$ & $\%$ & $n$ & $\%$ \\
\hline \multicolumn{5}{|c|}{ Edad (años) } \\
\hline $18-<28$ & 2 & 2,9 & 1 & 0,7 \\
\hline $28-<38$ & 2 & 2,9 & 11 & 8,2 \\
\hline $38-<48$ & 13 & 19,1 & 27 & 20,1 \\
\hline $48-<58$ & 17 & 25 & 38 & 28,4 \\
\hline $58-<68$ & 20 & 29,4 & 31 & 23,1 \\
\hline $68-<78$ & 9 & 13,2 & 18 & 13,4 \\
\hline$\geq 78$ & 5 & 7,4 & 8 & 6 \\
\hline \multicolumn{5}{|c|}{$I M C\left(\mathrm{~kg} / \mathrm{m}^{2}\right)$} \\
\hline Bajo peso & 5 & 7,4 & 5 & 3,7 \\
\hline Normal & 31 & 45,6 & 64 & 47,8 \\
\hline Sobrepeso & 27 & 39,7 & 49 & 36,6 \\
\hline Obesidad I & 5 & 7,4 & 15 & 11,2 \\
\hline Obesidad II & 0 & 0 & 1 & 0,7 \\
\hline \multicolumn{5}{|c|}{ Ocupación } \\
\hline Trabajadora & 39 & 57,4 & 64 & 47,8 \\
\hline Estudiante & 1 & 1,5 & 3 & 2,2 \\
\hline Ama de casa & 26 & 38,2 & 49 & 36,6 \\
\hline Jubilada & 1 & 1,5 & 8 & 6 \\
\hline Desempleada & 1 & 1,5 & 10 & 7,5 \\
\hline \multicolumn{5}{|c|}{ Estrato social } \\
\hline 1 & 28 & 41,2 & 77 & 57,5 \\
\hline 2 & 25 & 36,8 & 45 & 33,6 \\
\hline 3 & 15 & 22,1 & 12 & 9 \\
\hline \multicolumn{5}{|c|}{ Etapa hormonal } \\
\hline Premenopáusica & 23 & 33,8 & 42 & 31,3 \\
\hline Posmenopáusica & 45 & 66,2 & 92 & 68,7 \\
\hline \multicolumn{5}{|c|}{ Antecedentes obstétricos } \\
\hline Multípara & 56 & 82,4 & 102 & 76,1 \\
\hline Nulípara & 12 & 17,6 & 32 & 23,9 \\
\hline \multicolumn{5}{|c|}{ Fenotipo del cáncer de mama } \\
\hline Luminal A & 51 & 75 & 62 & 46,3 \\
\hline Luminal B & 8 & 11,8 & 25 & 18,7 \\
\hline Como mama sana & 0 & 0 & 3 & 2,2 \\
\hline HER 2 & 4 & 5,9 & 18 & 13,4 \\
\hline Basal like & 5 & 7,4 & 26 & 19,4 \\
\hline \multicolumn{5}{|c|}{ Estadio del cáncer de mama } \\
\hline I & 4 & 5,9 & 10 & 7,5 \\
\hline$\| \mathrm{A}$ & 21 & 30,9 & 49 & 36,6 \\
\hline IIB & 27 & 39,7 & 29 & 21,6 \\
\hline IIIA & 9 & 13,2 & 27 & 20,1 \\
\hline IIIB & 2 & 2,9 & 10 & 7,5 \\
\hline IIIC & 4 & 5,9 & 3 & 2,2 \\
\hline IV & 1 & 1,5 & 6 & 4,5 \\
\hline
\end{tabular}


Cuadro 2. Características de pacientes con hiperplasia endometrial tras el diagnóstico de cáncer de mama en relación con la duración del tratamiento con tamoxifeno

\begin{tabular}{|c|c|c|c|c|c|c|c|c|c|c|c|}
\hline \multicolumn{12}{|c|}{ Tiempo de uso tamoxifeno } \\
\hline & & \multicolumn{2}{|c|}{$\begin{array}{c}6-11 \text { meses } \\
(n=22)\end{array}$} & \multicolumn{2}{|c|}{$\begin{array}{c}1<2 \text { años } \\
\quad(n=17)\end{array}$} & \multicolumn{2}{|c|}{$\begin{array}{c}2<3 \text { años } \\
(n=15)\end{array}$} & \multicolumn{2}{|c|}{$\begin{array}{c}3 \geq 5 \text { años } \\
(n=5)\end{array}$} & \multicolumn{2}{|c|}{ No usó } \\
\hline \multirow{3}{*}{$\begin{array}{c}\text { Tipo de } \\
\text { hiperplasia }\end{array}$} & & $n$ & $\%$ & $n$ & $\%$ & $n$ & $\%$ & $n$ & $\%$ & $n$ & $\%$ \\
\hline & Con atipias & 9 & 40,9 & 6 & 35,3 & 8 & 53,3 & 2 & 40,0 & 0 & 0,0 \\
\hline & Sin atipias & 13 & 59,1 & 11 & 64,7 & 7 & 46,7 & 3 & 60,0 & 9 & 100,0 \\
\hline \multirow{3}{*}{$\begin{array}{c}\text { Comorbilidad } \\
\text { de la } \\
\text { hiperplasia }\end{array}$} & No afecta la vida & 8 & 36,4 & 6 & 35,3 & 4 & 26,7 & 2 & 40,0 & 5 & 55,6 \\
\hline & $\begin{array}{l}\text { Afecta } \\
\text { moderadamente }\end{array}$ & 7 & 31,8 & 7 & 41,2 & 5 & 33,3 & 1 & 20,0 & 4 & 44,4 \\
\hline & Genera incapacidad & 7 & 31,8 & 4 & 23,5 & 6 & 40,0 & 2 & 40,0 & 0 & 0,0 \\
\hline
\end{tabular}

El 86,7 \% de los casos y el 62,7 \% de los controles usaron tamoxifeno, encontrándose el uso del tamoxifeno asociado a hiperplasia endometrial (OR=3,90; IC95 \%: 1,7818,55). Las mujeres pertenecientes al estrato social 1 tuvieron 0,29 veces menos probabilidad de presentar hiperplasia endometrial que aquellas de un estrato más alto. Mientras que las mujeres que padecen de cáncer de mama de fenotipo luminal A presentaron 4,3 veces más riesgo de hiperplasia endometrial (IC95 \%: 1,53-11,94) que aquellas que presentan el fenotipo basal like (cuadro 3 ).

Tener receptores hormonales positivos individuales (RE o RP) o combinados (RE y RP) se identificó como factor asociado a hiperplasia endometrial, siendo mayor esta asociación cuando se portan de manera individual, pero siendo significativo solo para el receptor de estrógenos $(p<0,05)$. La mayoría de las pacientes presentó ambos receptores hormonales positivos (OR=4,26; IC95 \%: 1,77-10,23) (cuadro 3).

De los 68 casos, el 32,35 \% usó tamoxifeno en el intervalo de duración más corto evaluado (OR= 6,43, IC95 \%: 2,52-16,44), y 5,88\%, estuvieron en el intervalo de duración mayor $4-\geq 5$ años (OR=1,11, IC95 \%: 0,31-4,02). Con respecto a los controles el 37,1 \% no usó el medicamento; sin embargo entre quienes lo usaron el 77,48 \% lo hicieron por mas de un año.

Se exploraron varios modelos de regresión logística evaluando el papel del uso del tamoxifeno con la hiperplasia endometrial. En cada uno de ellos se realizaron los ajustes correspondientes por covariables asociadas a hiperplasia endometrial y en todos se observó asociación de las variables en estudio (cuadro 4). 
Enero - abril de 2021 - Pág 21

El 32,5\% de los casos, usó tamoxifeno en el período más corto evaluado y $5,8 \%$ en el período más largo. Con respecto a los controles el 62,7 \% no usó el medicamento.
Cuadro 3. Asociación de hiperplasia endometrial con factores demográficos y clínicos. Barranquilla, 2012-2017

\begin{tabular}{|c|c|c|c|c|c|c|c|}
\hline & \multirow{2}{*}{$\begin{array}{l}\text { Casos } \\
n=68\end{array}$} & \multirow{2}{*}{$\begin{array}{l}\text { Controles } \\
n=134\end{array}$} & \multirow{2}{*}{$O R$} & \multirow{2}{*}{\multicolumn{2}{|c|}{ IC $95 \%$}} & & \multirow{2}{*}{$p$} \\
\hline & & & & & & & \\
\hline \multicolumn{8}{|c|}{ Uso de tamoxifeno } \\
\hline No & 9 & 50 & Ref & & & & \\
\hline Sí & 59 & 84 & 3,9 & 1,78 & - & 8,55 & 0,000 \\
\hline \multicolumn{8}{|c|}{ Estrato social } \\
\hline 3 & 15 & 12 & Ref & & & & \\
\hline 1 & 28 & 77 & 0,29 & 0,12 & - & 0,70 & 0,005 \\
\hline 2 & 25 & 45 & 0,44 & 0,18 & - & 1,10 & 0,061 \\
\hline
\end{tabular}

Fenotipo del cáncer de mama

$\begin{array}{llllllll}\text { Basal like } & 5 & 26 & \text { Ref } & & & & \\ \text { Luminal A } & 51 & 62 & 4,28 & 1,53 & - & 11,94 & 0,002 \\ \text { Luminal B } & 8 & 25 & 1,66 & 0,48 & - & 5,78 & 0,311 \\ \text { Como mama sana } & 0 & 3 & 0 & 0,00 & - & 0,00 & 0,610 \\ \text { HER 2 } & 4 & 18 & 1,16 & 0,27 & - & 4,90 & 0,563\end{array}$

\section{Receptor hormonal}

$\begin{array}{llllllll}\text { Negativos } & 7 & 47 & \text { Ref } & & & & \\ \text { RE* }^{(+)} & 14 & 14 & 6,71 & 2,27 & - & 19,89 & 0,000 \\ \operatorname{RP}^{* *}(+) & 2 & 2 & 6,71 & 0,81 & - & 55,64 & 0,109 \\ \text { RE Y RP (+) } & 45 & 71 & 4,26 & 1,77 & - & 10,23 & 0,000\end{array}$

\section{Tiempo de uso del tamoxifeno (años)}

\begin{tabular}{llllllll}
\hline No uso & 9 & 50 & Ref & & & & \\
$<1$ & 22 & 19 & 6,43 & 2,52 & - & 16,44 & 0,000 \\
$1-<2$ & 17 & 20 & 4,72 & 1,81 & - & 12,33 & 0,001 \\
$2-<3$ & 15 & 13 & 6,41 & 2,29 & - & 17,91 & 0,000 \\
$3-<4$ & 1 & 12 & 0,46 & 0,05 & - & 4,01 & 0,421 \\
$4-\geq 5$ & 4 & 20 & 1,11 & 0,31 & - & 4,02 & 0,555 \\
\hline
\end{tabular}

*Receptor estrógenos. **Receptor progesterona. OR. Odds ratio
Cuadro 4. Modelos de regresión para hiperplasia relacionada con el uso de tamoxifeno

\begin{tabular}{ccc}
\hline Modelo & OR & IC $95 \%$ \\
\hline $1^{a}$ & 2,9 & $1,08-7,52$ \\
$2^{b}$ & 2,9 & $1,11-7,50$ \\
$3^{c}$ & 2,8 & $1,09-7,23$ \\
\hline
\end{tabular}

Ajustado por: Edad, IMC, estrato social, antecedentes obstétricos, estadio del cáncer, radioterapia quimioterapia, receptor hormonal y uso de tamoxifeno.

Ajustado por: Tiempo de uso de tamoxifeno, edad, receptor hormonal, estadio del cáncer, radioterapia, quimioterapia y comorbilidades.

'Ajustado por: Tiempo de uso de tamoxifeno, edad, receptor hormonal, estadio del cáncer, radioterapia y quimioterapia. 


\section{Discusión}

Este estudio corrobora la asociación entre el uso de tamoxifeno y el desarrollo de hiperplasia endometrial. Dicha asociación es similar a las publicadas por el estudio de Chalas et al. (11) que muestra un riesgo relativo de 2,06 para el desarrollo de hiperplasia endometrial en cualquier grupo de mujeres. Sin embargo, cabe destacar que este estudio corresponde a un ensayo clínico, en el cual las pacientes fueron seguidas cercanamente para lograr diagnóstico y monitoreo adecuado de cualquier cambio presentado a nivel ginecológico.

Los factores aquí estudiados y su relación con la hiperplasia en pacientes en tratamiento con tamoxifeno han sido previamente estudiados; entre ellos, se pueden destacar el hecho de ser posmenopáusica, nulípara e infértil; algunas comorbilidades como diabetes, hipertensión y obesidad también se asocian con un mayor riesgo, el tiempo de uso y la dosis acumulada también fueron factores de riesgo asociados a hiperplasias endometrial (12-14).

En cuanto al tiempo de uso, Dalbert et al., estudian 152 pacientes con cáncer de mama tratadas con tamoxifeno y encuentran que el tiempo de tratamiento influye desfavorablemente en la incidencia de afecciones endometriales, pues la mayor cantidad de pacientes con hiperplasia endometrial simple se presentaron luego de 24 meses de uso (15), lo que muestra gran diferencia con los resultados obtenidos en este estudio, donde el las participantes con menos de un año de uso obtuvo un probabilidad de desarrollar la hiperplasia seis veces mayor comparada con el resto de intervalos. Esto podría explicarse por dos razones: la primera es que muchas de

Este estudio corrobora la existencia de asociación entre el uso de tamoxifeno y el desarrollo de hiperplasia endometrial. las pacientes estuvieron en tratamiento con tamoxifeno de manera intermitente y los tiempos de uso totales fueron obtenidos de la suma total de los intervalos en los que estuvieron en tratamiento; en segundo lugar, una gran proporción de las pacientes que hicieron parte del grupo de casos estuvieron en tratamiento continuo en el tiempo indicado.

En el estudio de Swerdlow y Jones (16) el riesgo para desarrollar cáncer endometrial se incrementaba a medida que aumentaba el peso de las participantes, sobre todo en mujeres posmenopáusicas. En este estudio los puntos de corte para establecer IMC fueron similares, por lo que los resultados también lo fueron; sin embargo, la razón de disparidad (OR) obtenida no fue significativa. Otros estudios han mostrado resultados similares (17-19).

Aunque se ha evidenciado que la etapa hormonal (pre o posmenopáusicas) y los antecedentes obstétricos (ser multípara o nulípara) se relacionan con alteraciones del endometrio en pacientes tratadas con tamoxifeno (20), este estudio no encontró una asociación significativa de estas variables.

El estrato social más bajo demostró ser un factor protector significativo para el desarrollo del efecto adverso. Esto puede explicarse porque la mayoría de pacientes de los estratos más bajos tienen un menor acceso a los servicios del sistema de salud (21-23), con lo que podría pensarse que en esta población podría existir un subdiagnóstico de la hiperplasia endometrial asociada al tamoxifeno. De igual forma, la mayoría de las pacientes del estudio pertenecen a estratos bajos, dado que la institución participante cuenta con gran afluencia de usuarios de bajos recursos. 
Especial cuidado merecen aquellas mujeres con cáncer de mama con receptores hormonales positivos de estrógenos o con diagnóstico inmunohistoquímico de fenotipo Luminal $A$, puesto que presentan mayor riesgo que aquellas con fenotipo Basal like.
Otro de los resultados significativos del estudio fue la relación entre los distintos fenotipos reconocidos para cáncer de mama con el riesgo de desarrollo de hiperplasia endometrial, encontrándose asociación con el fenotipo Luminal A. Los tumores luminales han mostrado una mayor expresión de receptores hormonales positivos, por lo que está indicado tratamiento con un modulador selectivo de los receptores estrogénicos, como el tamoxifeno $(24,25)$, más usado a nivel nacional para el cáncer de mama, y del cual sí se encontró asociación con la hiperplasia endometrial. Actualmente, no se ha descrito un tratamiento específico para pacientes con tumores con el fenotipo en mención, lo cual podría ser una nueva aproximación práctica para dicho tratamiento $(26,27)$.

El uso de tamoxifeno es un factor de riesgo para el desarrollo de hiperplasia endometrial, incrementando 2,9 veces la probabilidad de padecerla en las usuarias, por lo cual sería ideal realizar seguimiento de las mujeres que reciban este tratamiento y aún más en las que presenten síntomas sugestivos de dicha complicación, como sangrado vaginal anormal o el manchado. Especial cuidado merecen aquellas mujeres con cáncer de mama con receptores hormonales positivos de estrógenos o con diagnóstico inmunohistoquímico de fenotipo Luminal A, puesto que presentan mayor riesgo que aquellas con fenotipo Basal like (25).

Este estudio mostró que no hay un gradiente biológico asociado al tiempo de uso del tamoxifeno, ya que el mayor riesgo se presentó en quienes fueron tratadas por tres años o menos, más concretamente en aquellas que lo usaron por 6 a 11 meses. Sin embargo, sí se relacionó una mayor duración del tratamiento con una mayor proporción en la generación de hiperplasia endometrial con atipias. Por lo mencionado, el médico debe sopesar cuidadosamente la relación riesgo-beneficio, pues a pesar de ser el tamoxifeno un efectivo medicamento de primera línea para el cáncer de mama, puede generar complicaciones incapacitantes y que disminuyan la calidad de vida.

Una potencial limitación de este estudio se relaciona con utilizar una base de datos proporcionada por un centro oncológico en donde el tratamiento estándar es el uso de tamoxifeno en la mayoría de mujeres con cáncer de mama que son elegibles para el uso del medicamento. Por lo tanto, efectos del uso del medicamento serán frecuentes es las pacientes evaluadas en el estudio, sin que estos exacerben el metabolismo normal del mismo en el organismo.

\section{Bibliografía}

1. International Agency for Research on Cancer (IARC). Global cancer statistics 2018 -GLOBOCAN 2018-. Graph production: IARC (http://gco.iarc.fr/today) World Health Organization. Lyon, France. 2018.

2. Terán Dávila J, Teppa Garrán AD. Moduladores selectivos de los receptores estrogénicos (SERMs): Bioquímica, farmacología y aplicación clínica en ginecología. Ginecol Obstet Mex. 2005;73(8):424-35.

3. Yang G, Nowsheen S, Aziz K, Georgakilas AG. Toxicity and adverse effects of Tamoxifen and other anti-estrogen drugs. Pharmacol Ther. 2013;139(3):392-404. Available from: http://www.ncbi.nlm.nih.gov/pubmed/23711794 
4. Chicaíza L, Gamboa, O García, M. Tamoxifen vs. Inhibidores de aromatasa para cáncer de mama: análisis de costo efectividad para Colombia. Repositorio Institucional Universidad Nacional. 2008;(2):1-11.

5. Killackey MA, Hakes TB, Pierce VK. Endometrial adenocarcinoma in breast cancer patients receiving antiestrogens. Cancer Treat Rep. 1985;(69):237-8.

6. Lumachi F, Brunello a, Maruzzo M, Basso U, Basso SMM. Treatment of estrogen receptor-positive breast cancer. Curr Med Chem. 2013;20(5):596-604.

7. Barnard ME, Boeke CE, Tamimi RM. Established breast cancer risk factors and risk of intrinsic tumor subtypes. Biochim Biophys Acta. 2015;1856(1):73-85. Available from: http://www.ncbi.nlm.nih.gov/pubmed/26071880

8. Yeung NCY, Zhang Y, Ji L, Lu G, Lu Q. Correlates of sexual quality of life among husbands of Chinese breast cancer survivors. Eur J Oncol Nurs. 2019;(40):63-70. Available from: http://www.ncbi.nlm.nih.gov/pubmed/31229208

9. Person H, Guillemin F, Conroy T, Velten M, Rotonda C. Factors of the evolution of fatigue dimensions in patients with breast cancer during the 2 years after surgery. Int J cancer. 2019; (0):0-00; Available from: http://www.ncbi.nlm.nih.gov/ pubmed/31228259

10. Lam WWT, Soong I, Yau TK, Wong KY, Tsang J, Yeo W, et al. The evolution of psychological distress trajectories in women diagnosed with advanced breast cancer: a longitudinal study. Psychooncology. 2013;22(12):2831-9. Available from: http:// www.ncbi.nlm.nih.gov/pubmed/24038545

11. Chalas E, Costantino JP, Wickerham DL, Wolmark N, Lewis GC, Bergman C, et al. Benign gynecologic conditions among participants in the Breast Cancer Prevention Trial. Am J Obstet Gynecol. 2005;(192):1230-9.

12. Chandra V, Kim JJ, Benbrook DM, Dwivedi A, Rai R. Therapeutic options for management of endometrial hyperplasia. J Gynecol Oncol. 2016;27(1):1-25.

13. Lauby-Secretan B, Dossus L, Marant-Micallef C, His M. Obesity and Cancer. Bull Cancer. 2019;106(7-8):635-646; Available from: http://www.ncbi.nlm.nih.gov/ pubmed/31227175

14. Cuzick J, Sestak I, Baum M, Buzdar A, Howell A, Dowsett M, et al. Effect of anastrozole and tamoxifen as adjuvant treatment for early-stage breast cancer: 10year analysis of the ATAC trial. Lancet Oncol [Internet]. 2010 Dec;11(12):1135-41. Available from: http://www.ncbi.nlm.nih.gov/pubmed/21087898

15. Dalbert D, Rodríguez M, Figueredo A, Mural J, Bartt O, Subiela R et al. Tamoxifeno y afecciones endometriales en pacientes con cáncer de mama. Medicina (Buenos Aires). 2013;73(2):97-103.

16. Swerdlow AJ, Jones ME. Tamoxifen treatment for breast cancer and risk of endometrial cancer: a case-control study. J Natl Cancer Inst. 2005;97(5):375-84. 
17. Semiglazov VF, Maksimov SI, Bulgatova EA, Meshkova IE, Chepik OF, Berstein LM. Risk of endometrial hyperplasia and carcinoma in breast cancer patients receiving adjuvant tamoxifen. Vopr Onkol. 2003;49(2):198-204. Available from: http://www.ncbi.nlm.nih.gov/pubmed/12785205

18. Yin L, Li J, Wei Y, Ma D, Sun Y, Sun Y. Primary ovarian small cell carcinoma of pulmonary type with coexisting endometrial carcinoma in a breast cancer patient receiving tamoxifen: A case report and literature review. Medicine (Baltimore). 2018;97(23):e10900. Available from: http://www.ncbi.nlm.nih.gov/pubmed/29879027

19. Chandra V, Kim JJ, Benbrook DM, Dwivedi A, Rai R. Therapeutic options for management of endometrial hyperplasia. J Gynecol Oncol. 2016;27(1):e8. Available from: http://www.ncbi.nlm.nih.gov/pubmed/26463434

20. Jeon J, Kim SE, Lee D-Y, Choi D. Factors associated with endometrial pathology during tamoxifen therapy in women with breast cancer: a retrospective analysis of 821 biopsies. Breast Cancer Res Treat. 2020;179(1):125-30. Available from: http://www.ncbi.nlm.nih.gov/pubmed/31541382

21. Winters S, Martin C, Murphy D, Shokar NK. Breast Cancer Epidemiology, Prevention, and Screening. Prog Mol Biol Transl Sci. 2017;(151):1-32. Available from: http://www.ncbi.nlm.nih.gov/pubmed/29096890

22. Piñeros M, Sánchez R, Perry P, García O, Ocampo R, Cendales R. Demoras en el diagnóstico y tratamiento de mujeres con cáncer de mama en Bogotá, Colombia. Salud Publica Mex. 2011;53(6):478-85.

23. Sánchez G, Gustavo C, Estupiñan C. Factores asociados con el tratamiento oportuno de mujeres con cáncer de mama apoyadas por una organización no gubernamental en Bogotá. Biomédica. 2015;(35):505-12.

24. Ministerio de Salud y Protección Social. Guía de Práctica Clínica (GPC) para la detección temprana, tratamiento integral, seguimiento y rehabilitación de pacientes con cáncer de mama. Sistema de Seguridad Social, Colombia, 2013. Guía No. 19

25. Zhao SG, Chen WS, Das R, Chang SL, Tomlins SA, Chou J, et al. Clinical and Genomic Implications of Luminal and Basal Subtypes Across Carcinomas. Clin Cancer Res. 2019;25(8):2450-7. Available from: http://www.ncbi.nlm.nih.gov/ pubmed/30573691

26. Gao JJ, Swain SM. Luminal A Breast Cancer and Molecular Assays: A Review. Oncologist [Internet]. 2018;23(5):556-65. Available from: http://www.ncbi.nlm. nih.gov/pubmed/29472313

27. Stöger $H$. Are the new breast cancer subtypes (luminal $A, B$ etc.) of practical impact? Yes. Dtsch Med Wochenschr. 2013;138(41):20-96. Available from: http:// www.ncbi.nlm.nih.gov/pubmed/24085364 